\title{
Evaluación De Tres Niveles De Mananos Oligosacáridos (Sacharomices Cerevisae) En Los Parámetros Productivos Y Salud Intestinal En Pollos De Engorde En El Cantón Babahoyo, Provincia De Los Ríos, Ecuador
}

\author{
Mvz. Ricardo Zambrano Moreira \\ Mvz. Juan Carlos Gómez Villalva \\ Dr. Mvz. Johns Rodríguez Álava \\ Mvz. Hugo Alvarado Álvarez. \\ Dr. Mvz. Luis Quezada Gallardo \\ Dr. Mvz. William Filian Hurtado \\ Dr. Mvz. Edison Ponce Cepeda \\ Universidad Técnica de Babahoyo \\ PhD. Juan Avellaneda Cevallos \\ Universidad Estatal de Quevedo
}

doi: 10.19044/esj.2017.v13n12p24 URL:http://dx.doi.org/10.19044/esj.2017.v13n12p24

\begin{abstract}
The objective of this research was to evaluate different levels of mannans oligosaccharides (MOs) in productive parameters and intestinal health in broilers. Two hundred and forty one-day-old broiler chickens (Ross 308 line) were used during a 6-week experimental period. A completely randomized design with four treatments and six replicates was used. The evaluated levels were $0.0 \mathrm{~kg} \mathrm{t}-1$ (T0); $0.5 \mathrm{~kg} \mathrm{t}-1$ (T 1); $1 \mathrm{~kg} \mathrm{t}-1$ (T2) and 1.5 $\mathrm{kg}$ t-1 (T3). For the mean comparisons, a Tukey test $(\mathrm{P} \leq 0.05)$ was used. The source of oligosaccharide mannans derived from the cell wall of Saccharomyces cerevisiae and it was added to the commercial balanced feed. Productive parameters, intestinal villi and economic performance (costbenefit) were evaluated. The data was analyzed with the statistical program SAS V9 (2012). At 42 days of the test, T2 reached the highest average weight of $2455.48 \mathrm{~g}$. For feed consumption, the mean average for T0, T1, T3 and $\mathrm{T} 2$ were $4342.63 \mathrm{~g}, 4304.5 \mathrm{~g}, 4254.32 \mathrm{~g}$ and $4216.80 \mathrm{~g}$ respectively. T2 had higher weight gain compare to the control group, 535.70 and $388.84 \mathrm{~g}$, respectively. Feed conversion was higher for T2 (1.71) compared to the control group (1.85). T1 obtained had a carcass yield of $75.89 \%$. Mortality
\end{abstract}


did not show significant differences $(\mathrm{P} \geq 0.05)$. Length and width $(\mu \mathrm{m})$ of the intestinal villi were different $(\mathrm{P} \leq 0.05)$ among treatments, at 21 days of, $\mathrm{T} 3$ showed the longest $(1939.74 \mu \mathrm{m})$ and widest $(326.03 \mu \mathrm{m})$ measures at the duodenum site. The economic analysis, all the treatments were profitable results, although $\mathrm{T} 1$ had the highest cost-benefits $(\$ 1.30)$.

Keywords: Mannose oligosaccharides, intestinal health, broilers, intestinal villi

\section{Resumen}

El objetivo de esta investigación fue evaluar diferentes niveles de mananos oligosacáridos (MOs) en los parámetros productivos y salud intestinal en pollos de engorde. Se utilizaron 240 pollos de la línea Ross 308, desde 1 a 42 días de edad, fueron asignados en un diseño completamente al azar, con cuatro tratamientos y seis repeticiones, diez pollos por unidad experimental. Los niveles evaluados: $0.0 \mathrm{~kg} \mathrm{t}^{-1}\left(\mathrm{~T}_{0}\right) ; 0.5 \mathrm{~kg}$ $\mathrm{t}^{-1}\left(\mathrm{~T}_{1}\right) ; 1 \mathrm{~kg} \mathrm{t}^{-1}\left(\mathrm{~T}_{2}\right)$ y $1.5 \mathrm{~kg} \mathrm{t}^{-1}\left(\mathrm{~T}_{3}\right)$. Para las comparaciones de los promedios se utilizó la prueba de Tukey para $\mathrm{P} \leq 0.05$. En el alimento balanceado comercial se adicionó mananos oligosacáridos derivados de la pared celular de Saccharomyces Cerevisiae. Se evaluaron parámetros productivos, vellosidades intestinales y rendimiento económico (costobeneficio). Los datos se analizaron con el programa estadístico SAS V9 (2012). A los 42 días del ensayo, el $\mathrm{T}_{2}$ alcanzó el mayor peso promedio de $2455.48 \mathrm{~g}$; en el consumo de alimento, se obtuvieron promedios de 4342.63 g, $4304.5 \mathrm{~g}, 4254.32 \mathrm{~g}$ y $4216.80 \mathrm{~g}$ para los T0, T1, T3 y T2, en su orden, el $\mathrm{T}_{2}$ alcanzó un incremento peso de $535.70 \mathrm{~g}$, superior al testigo (388.84 g); la conversión alimenticia fue mejor para el $\mathrm{T}_{2}$, con $1.71(\mathrm{~g} / \mathrm{g})$ comparada al control (1.85 g/g); el $\mathrm{T}_{1}$ obtuvo rendimiento a la canal de $75.89 \%$; la mortalidad no mostro diferencias significativas $(\mathrm{P} \geq 0.05)$; la longitud $\mathrm{y}$ ancho $(\mu \mathrm{m})$ de las vellosidades intestinales presentaron diferencia $(\mathrm{P} \leq 0.05)$ entre los tratamientos, a los 21 días de edad de los pollos, el $\mathrm{T}_{3}$ alcanzó mayor longitud $(1939.74 \mu \mathrm{m})$ y ancho $(326.03 \mu \mathrm{m})$ a nivel del duodeno; respecto al análisis económico, todos los tratamientos obtuvieron resultados rentables, destacándose el $\mathrm{T}_{1}$ registrando costos- beneficios de $\$ 1.30$

Palabras claves: Mánanos oligosacáridos, salud intestinal, pollos de engorde, vellosidades intestinales

\section{Introducción}

En las últimas décadas la avicultura ha alcanzado grandes avances y esto se debe principalmente a la acción conjunta entre genética, nutrición, sanidad y manejo. La producción avícola paso de ser una industria familiar a 
uno de los componentes más importantes de los negocios agropecuarios mundiales (Winder, 2004). En muchos sistemas productivos se ha hecho uso de antibióticos promotores del crecimiento (APC) en los concentrados para pollos de engorde, con el fin de controlar la microbiota entérica patógena (Gaggia, Marttarelli y Biavat, 2010). Sin embargo, su empleo continuado generó preocupaciones a los consumidores debido a la cantidad de residuos que quedan en la carne de los animales y en sus productos, sin contar con la resistencia generada en algunas cepas bacterianas por la administración continuada.

A partir de este momento se inició una búsqueda de alternativas de remplazo a los antibióticos promotores de crecimiento, y se postularon los probióticos como una alternativa viable, por ser un producto natural y sin riesgo para la salud del consumidor (Gutierrez, Montoya, \& Velez, 2013). Son muchos los factores que pueden influenciar en el desempeño del tracto gastrointestinal: los estímulos inmunitarios, el medio ambiente, la nutrición, el tipo y la calidad de los ingredientes de la ración, las toxinas, el equilibrio de la microflora, las secreciones endógenas, la motilidad y los aditivos (Torrealba, 2007). Los cambios de la morfología intestinal, tales como el acortamiento de las vellosidades intestinales y las criptas más profundas se han asociado con la presencia de toxinas; el acortamiento de las vellosidades intestinales reduce la superficie de absorción de nutrientes (Newman, 2002).

Zoetis (2013) indica, un tracto digestivo saludable, con su población microbial asociada balanceada, y adecuadas secreciones enzimáticas digestivas, es esencial para obtener un buen desempeño acorde con el potencial genético del pollo. El tracto gastrointestinal es una comunidad diversa de bacterias, hongos, protozoos y virus (microbiotica intestinal), el desarrollo de esta comunidad se inicia en el nacimiento del ave y las bacterias se adquieren del medio ambiente (Bailey, 2013).

Las industrias productoras de alimentos para monogástricos están continuamente investigando alternativas para sustituir los antibióticos promotores de crecimientos (APC). En el mercado existen diferentes aditivos utilizados en la alimentación de aves, se encuentran los ácidos orgánicos y prebióticos, que logran obtener parámetros productivos en pollos equiparables a los antibióticos (López, 2010).

Los prebióticos representan una alternativa tecnológica para mejorar la eficacia, definidos como un alimento o nutriente que pasa por el intestino delgado y es fermentado por la micro flora endógena (Waldroup, Fristtis y Oviedo, 2003). Los productos comerciales de MOs pueden reducir los patógenos entéricos, modular la respuesta inmunológica de los animales y mejorar la integridad de la mucosa intestinal (Torrealba, 2007). Los MOs mejoran el desempeño y la salud de las aves, principalmente, promoviendo la salud del tracto gastrointestinal. De acuerdo con varios investigadores, los 
MOs no solamente afectan los mecanismos de defensa no inmunológicos del tracto gastrointestinal, sino que funcionan a través de la modulación de las protecciones inmunológicas. Estas propiedades parecen ser factores muy importantes, que se han visto cuando se agrega MOs a las dietas animales (Ortiz, 2005).

Los sistemas de producción intensiva han hecho de la carne de pollo uno de los alimentos más consumidos en el mundo, aporta con aminoácidos esenciales, vitaminas y minerales en la alimentación humana (Narváez, 2008). La avicultura es una de las de mayor importancia dentro del sector agropecuario ecuatoriano, por su participación en la actividad económico y social, se estima que equivale al 13\% del PIB Agropecuario y al $4.6 \%$ del PEA así como por el aporte a la seguridad alimentaria, generación de empleos directos e indirectos.

En Ecuador la industria avícola comienza en la década de los 60 y en los últimos años, la producción se ha incrementado por la introducción de razas mejoradas, de alta producción de carne y huevo (Duque, 2010). Según la Corporación nacional de avicultores del Ecuador (CONAVE), la industria avícola es un sector de enorme importancia socio-económica y constituyen una de las principales fuentes de proteína de origen animal para los ecuatorianos, participó con el 13\% del Producto interno bruto (PIB) agropecuario en el año 2012, de los cuales la producción de carne de pollo representa el 5.6\%. Esta actividad impacta positivamente en el desarrollo de las zonas rurales del país (CONAVE, 2012). Pichincha y Tungurahua las provincias de mayor producción en la Sierra, Manabí y Guayas en la Costa (Duque, 2010).

La investigación realizada tuvo como objetivo evaluar el efecto de tres niveles $\left(0.5, \quad 1.0 \quad\right.$ y $\left.1.5 \quad \mathrm{~kg}^{-1}\right)$ de mánanos oligosacáridos (Saccharomyces Cerevisiae) en los parámetros productivos y salud intestinal en pollos Broiler en época húmeda en la zona de Babahoyo, mediante la identificación del nivel de MOs de mayor rendimiento y la medición de la longitud y grosor de las vellosidades intestinales en el ID, y la determinación del costo beneficio.

\section{Materiales y métodos}

\section{Ubicación y Descripción del Área Experimental}

La presente investigación se realizó en la Granja Experimental San Pablo de la Universidad Técnica de Babahoyo, ubicada en el Km 7 1/2 de la vía Montalvo de la provincia de Los Ríos, con las siguientes coordenadas: $01^{\circ} 49^{\prime}$ de latitud Sur y $79^{\circ} 32^{\prime}$ de latitud Oeste. La zona presenta una altura de $8 \mathrm{msnm}$, temperatura promedio anual de $25,6{ }^{\circ} \mathrm{C}$, humedad relativa promedio anual $82 \%$, precipitación medio anual $1569 \mathrm{~mm}$, heliófania 998.2 horas/luz/año, evaporación $720.5 \mathrm{~mm}$, suelo limo arcilloso. 


\section{Materiales}

Pollos broiler, manano oligosacáridos de origen de levadura de cerveza, vellosidades intestinales del intestino delgado.

\section{Factores Estudiados}

Variables Independientes: Comportamiento productivo del pollo Longitud y grosor de las vellosidades

Variable Dependiente: Dosis de manano oligosacárido.

\section{Métodos}

Se emplearon métodos de inducción, deducción, análisis, síntesis y método práctico experimental.

\section{Tratamientos}

Los factores considerados en la investigación fueron niveles de mánanos oligosacárido $\left(\mathrm{kg} \mathrm{t}^{-1}\right)$ de; 0.50: 1.00 y 1.50 .

\section{Diseño experimental}

El diseño experimental utilizado fue completamente al Azar (D.C.A), con 4 tratamientos, 6 repeticiones por tratamiento y 10 pollos en cada unidad experimental, distribuidos al azar.

Tabla 1. Esquema del experimento basado en el estudio de la adición de los niveles de MOs

\begin{tabular}{cccc}
\hline Dosis $\mathrm{Kg} \mathrm{t}^{-1}$ & (MOs) Tratamiento & $\begin{array}{c}\text { Tamaño Unidad } \\
\text { experimental }\end{array}$ & $\begin{array}{c}\text { Total Unidad } \\
\text { experimental por } \\
\text { tratamiento }\end{array}$ \\
\hline 0.00 & Bc mas MOs & 10 & 60 \\
0.50 & Bc mas MOs & 10 & 60 \\
1.00 & Bc mas MOs & 10 & 60 \\
1.50 & Bc mas MOs & 10 & 60 \\
\hline \multicolumn{4}{c}{ Bc= balanceado comercial }
\end{tabular}

\section{Análisis Funcional}

Todas las variables fueron sometidas al análisis de varianza y para determinar las diferencias estadísticas de rangos múltiples se utilizaron la prueba de Tukey al 5\% de significancia.

Tabla 2. Análisis de varianza (ADEVA)

\begin{tabular}{ccc}
\hline Fuente de variación & & Grados de libertad \\
\hline Tratamientos & $\mathrm{t}-1$ & $4-1=3$ \\
Error & $\mathrm{t}(\mathrm{r}-1)$ & $4(6-1)=20$
\end{tabular}




\section{Manejo del ensayo}

Para el desarrollo del experimento se realizaron las labores de preparación del galpón, se dividió en cubículos y aleatoriamente se asignaron los tratamientos y repeticiones. Se adicionó al pienso comercial las siguientes dosis: $0.50,1.00$ y $1.50 \mathrm{~kg} \mathrm{t}^{-1}$ de MOs para las fases de crecimiento y acabado. La investigación, tuvo una duración de 42 dias, los parámetros productivos fueron registrados cada 7 dias. A los 21, 35 y 42 dias de edad, se enviaron al laboratorio; 2 muestras del intestino delgado por tratamiento, para la medición de altura y grosor de las vellosidades.

\section{Datos evaluados}

Los indicadores evaluados fueron: peso corporal, consumo de alimento, conversión alimenticia, ganancia de peso, mortalidad, rendimiento a la canal, longitud y grosor de vellosidades.

\section{Resultados y discusión}

En la Tabla 3 se presentan las comparaciones de las medias de los pesos corporales en los tratamientos, se puede observar que en la primera y quinta semana no hubo diferencias $(\mathrm{P} \geq 0.05)$, mientras que en la segunda, tercera, cuarta y sexta semana se observó un comportamiento contrario $(\mathrm{P} \leq$ 0.05). Estos resultados fueron menores a los obtenidos por Gómez (2012), quien evaluó 2 niveles de mananos oligosacáridos como aditivo en el alimento en pollos Broilers, obteniendo pesos promedio de 2728,10 (0.5 g $\left.\mathrm{kg}^{-1}\right)$ y $2768.10\left(1 \mathrm{~g} \mathrm{~kg}^{-1}\right)$. Por su parte Benítez y Reina (2007) estudiaron el efectos de dos fuentes comerciales de mánanos oligosacáridos; Bio-Mos IFC y Safmannan, en niveles de 0.0 y $0.5 \mathrm{~g} \mathrm{~kg}^{-1}$ de alimento; observando que las aves del Bio-Mos IFC ( $\left.0.5 \mathrm{~g} \mathrm{Kg}^{-1}\right)$ presentaron mayor peso corporal (2497.4; $\mathrm{P} \leq 0.05$ ) que los demás tratamientos; con valores de 2440.3 (testigo), 2495.4 ( Bio-Mos, $0.0 \mathrm{~g} \mathrm{~kg}^{-1}$ ), 2429.90 (Safmannan, 0.5 $\mathrm{g} \mathrm{kg}^{-1}$ ) y 2405.60 (Safmannan, $0.0 \mathrm{~g} \mathrm{~kg}^{-1}$ ) a los 42 días de edad en pollos de engorde.

Tabla 3. Efectos de mánanos oligosacáridos sobre el pesos corporal (g/ave) en los tratamientos.

\begin{tabular}{|c|c|c|c|c|c|c|}
\hline \multirow{2}{*}{ Semana } & \multicolumn{4}{|c|}{ Tratamientos } & \multirow{2}{*}{ E.E.M. } & \multirow{2}{*}{$\mathrm{P} \geq$} \\
\hline & T0 & $\mathrm{T} 1$ & $\mathrm{~T} 2$ & $\mathrm{~T} 3$ & & \\
\hline 1 & $165.24 \mathrm{a}$ & $168.36 \mathrm{a}$ & $166.80 \mathrm{a}$ & $167.34 \mathrm{a}$ & 1.28 & 0.7641 \\
\hline 2 & $421.07 \mathrm{ab}$ & $416.15 b c$ & $426.40 \mathrm{a}$ & $410.315 c$ & 1.10 & 0.0010 \\
\hline 3 & $799.78 \mathrm{~b}$ & $817.49 \mathrm{ab}$ & $835.24 a$ & $802.27 b$ & 3.08 & 0.0003 \\
\hline 4 & $1308.12 \mathrm{a}$ & $1327.87 \mathrm{~b}$ & $1323.60 \mathrm{a}$ & $1290.93 a$ & 7.95 & 0.2053 \\
\hline 5 & $1954.09 a$ & $1950.31 \mathrm{a}$ & $1919.77 \mathrm{a}$ & $1916.42 \mathrm{a}$ & 17.00 & 0.6812 \\
\hline 6 & $2342.93 \mathrm{c}$ & $2380.18 b c$ & $2455.48 \mathrm{a}$ & $2392.57 b$ & 7.34 & 0.0001 \\
\hline
\end{tabular}


Promedios con letras iguales no difieren estadísticamente, según Tukey $(\mathrm{P} \leq 0.05)$. EEM $=$ error estándar de la media; $\mathrm{CV} \%=$ coeficiente de variación; .T0 $\mathrm{T} 0$ Testigo; $\mathrm{T} 1=0,5 \mathrm{~kg} \mathrm{t}^{-}$

${ }^{1} \mathrm{MOs} ; \mathrm{T} 2=1 \mathrm{~kg} \mathrm{t}^{-1} \mathrm{MOs} ; \mathrm{T} 3=1,5 \mathrm{~kg} \mathrm{t}^{-1}$ MOs.

\section{Consumo de alimento}

Los resultados obtenidos en el consumo de alimento semanal se muestran en la tabla 4, en la misma se puede evidenciar que en la semana uno y dos no hubo diferencia significativa ( $\mathrm{P} \geq 0.05)$, pero si en la semana tres, cuatro, cinco y seis entre los tratamientos. El menor consumo lo obtuvo el tratamiento dos (4216.80 g), comparado con el testigo que alcanzo mayor consumo (4342. $63 \mathrm{~g}$ ). Estos resultados son similares a los obtenidos por Vásquez y Fernández (2010), quienes evaluaron tres dosis de Actigen en pollos de engorde en alimento a los 42 días de edad, obteniendo pesos promedios de $4331.5 \mathrm{~g}$ (Actigen, 1-21 días $400 \mathrm{~g}, 22-42$ días $400 \mathrm{~g}$ ), 4301.5 (Actigen, 1-21 días $400 \mathrm{~g}, 22-42$ días $200 \mathrm{~g} \mathrm{t}^{-1}$ ) y $4341.6 \mathrm{~g}$ (Actigen, 1-21 días $200 \mathrm{~g}, 22-42$ días $\left.200 \mathrm{~g} \mathrm{t}^{-1}\right)$. Y fueron mayores a los reportados por Andrade y Ayala (2011), que evidenciaron consumo de alimento (g) a los 42 días de 3655.0, 3656.9 y $3660.5 \mathrm{~g}$, cuando utilizaron mánanos oligosacárido en niveles de $0.5,1.0, \mathrm{y} 1.5 \mathrm{~g} \mathrm{~kg}^{-1}$ de alimento.

Tabla 4. Efectos de los niveles de mánanos oligosacáridos sobre el consumo de alimento

(g) en pollos de engorde

\begin{tabular}{ccccccc}
\hline \multirow{2}{*}{ Semana } & \multicolumn{5}{c}{ Tratamientos } & \multirow{2}{*}{$\mathrm{P} \geq$} \\
\cline { 2 - 5 } & $\mathrm{T} 0$ & $\mathrm{~T} 1$ & $\mathrm{~T} 2$ & $\mathrm{~T} 3$ & E.E.M. & \\
\hline 1 & $139.75 \mathrm{a}$ & $140.92 \mathrm{a}$ & $138.45 \mathrm{a}$ & $139.57 \mathrm{a}$ & 0.69 & 0.1026 \\
2 & $343.60 \mathrm{a}$ & $353.60 \mathrm{a}$ & $349.87 \mathrm{a}$ & $330.88 \mathrm{a}$ & 6.47 & 0.1011 \\
3 & $583.25 \mathrm{ab}$ & $610.90 \mathrm{a}$ & $556.00 \mathrm{~b}$ & $584.77 \mathrm{a}$ & 7.03 & 0.0004 \\
4 & $939.28 \mathrm{a}$ & $884.57 \mathrm{~b}$ & $892.35 \mathrm{~b}$ & $858.07 \mathrm{c}$ & 4.97 & 0.0001 \\
5 & $1158.85 \mathrm{a}$ & $1129.82 \mathrm{ab}$ & $1120.38 \mathrm{~b}$ & $1154.98 \mathrm{a}$ & 7.95 & 0.0001 \\
6 & $1178.28 \mathrm{a}$ & $1184.58 \mathrm{ab}$ & $1159.87 \mathrm{~b}$ & $1186.05 \mathrm{a}$ & 4.60 & 0.0001 \\
Promedio & $4342.63 \mathrm{a}$ & $4304.57 \mathrm{ab}$ & $4216.80 \mathrm{c}$ & $4254.32 \mathrm{bc}$ & 9.75 & 0.0001 \\
\hline
\end{tabular}

Promedios con letras iguales no difieren estadísticamente, según Tukey $(\mathrm{P} \leq 0.05)$. EEM $=$ error estándar de la media; $\mathrm{CV} \%=$ coeficiente de variación; .T0 $\mathrm{T}$ Testigo; $\mathrm{T} 1=0,5 \mathrm{~kg} \mathrm{t}^{-}$

${ }^{1} \mathrm{MOs} ; \mathrm{T} 2=1 \mathrm{~kg} \mathrm{t}^{-1} \mathrm{MOs} ; \mathrm{T} 3=1,5 \mathrm{~kg} \mathrm{t}^{-1} \mathrm{MOs}$.

\section{Conversión alimenticia}

En la tabla 5, se presentan los resultados obtenidos durante la investigación, en lo referente a la conversión, se registraron diferencias significativas $(\mathrm{P} \leq 0.05)$ entre la semanas tres y seis, por el contrario no se presentaron diferencias $(\mathrm{P} \geq 0.05)$ en las semanas uno, dos, cuatro y cinco. La mejor conversión alimenticia la obtuvo el tratamiento dos a los 42 días de1.71b, seguida de T3 con 1.77b, T1 de 1,80b y T0 de 1,85a. Estos resultados fueron similares a los obtenido por Nicoletti, Flores, Terraes y Kuttel (2010), analizaron parámetros productivos y morfológicos en pollos 
parrilleros suplementados con extracto de pared de levadura (Saccharomyces cerevisiae), y ácidos orgánicos, alcanzando conversión alimenticia de 1.7 con $1.5 \mathrm{~kg} \mathrm{t}^{-1}$ de alimento a los 42 días de edad de los pollos, siendo mejores a los reportados por Gonzales, Piad y Reyes (2013), con índice de conversión alimenticia de 1.89, al evaluar derivado de paredes celulares de levadura Saccharomyces cerevisiae (PCL-Glucano) al 0.10\% PCL-Glucano, a los 42 días de edad. Resultado semejante obtuvo Nicolalde (2009), el cual encontró conversión alimenticia de 1.73 al evaluar mánanos oligosacárido $\left(1.0 \mathrm{~kg} \mathrm{t}^{-1}\right.$ de alimento) y ácido orgánico en los parámetros productivos en pollos de engorde, a los 49 día edad.

Tabla 5. Efectos de los niveles de mánanos oligosacáridos sobre la conversión alimenticia $(\mathrm{g} / \mathrm{g})$ en pollos de engorde

\begin{tabular}{|c|c|c|c|c|c|c|}
\hline \multirow{2}{*}{ Semanas } & \multicolumn{4}{|c|}{ Tratamientos } & \multirow{2}{*}{ E.E.M. } & \multirow{2}{*}{$\mathrm{P} \geq$} \\
\hline & T0 & $\mathrm{T} 1$ & $\mathrm{~T} 2$ & $\mathrm{~T} 3$ & & \\
\hline 1 & $0.84 \mathrm{a}$ & $0.83 \mathrm{a}$ & $0.83 a$ & $0.83 a$ & 0.006 & 0.6849 \\
\hline 2 & $0.81 \mathrm{a}$ & $1.18 \mathrm{a}$ & $1.14 \mathrm{a}$ & $1.14 \mathrm{a}$ & 0.138 & 0.3825 \\
\hline 3 & $1.33 \mathrm{a}$ & $1.35 \mathrm{a}$ & $1.25 \mathrm{~b}$ & $1.31 \mathrm{a}$ & 0.007 & 0.0001 \\
\hline 4 & $1.53 \mathrm{a}$ & $1.50 \mathrm{a}$ & $1.46 \mathrm{a}$ & $1.48 \mathrm{a}$ & 0.010 & 0.0704 \\
\hline 5 & $1.62 \mathrm{a}$ & $1.60 \mathrm{a}$ & $1.59 \mathrm{a}$ & $1.60 \mathrm{a}$ & 0.013 & 0.8721 \\
\hline 6 & $1.85 \mathrm{a}$ & $1.80 \mathrm{~b}$ & $1.71 \mathrm{~b}$ & $1.77 \mathrm{~b}$ & 0.009 & 0.0001 \\
\hline
\end{tabular}

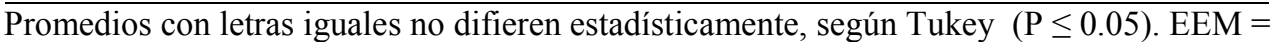
error estándar de la media; $\mathrm{CV} \%=$ coeficiente de variación; .T0= Testigo; $\mathrm{T} 1=0,5 \mathrm{~kg} \mathrm{t}^{-}$

${ }^{1} \mathrm{MOs} ; \mathrm{T} 2=1 \mathrm{~kg} \mathrm{t}^{-1} \mathrm{MOs} ; \mathrm{T} 3=1,5 \mathrm{~kg} \mathrm{t}^{-1} \mathrm{MOs}$.

\section{Incremento de peso}

Las medias generales de los incrementos de pesos se presentan en la tabla 6, en la misma se puede observar que en segunda, tercera y sexta semana presentaron diferencia significativa $(\mathrm{P} \leq 0.05)$, pero en la primera, cuarta y quinta semana no se presentó significancia $(\mathrm{P} \geq 0.05)$. A la sexta semana el mayor incremento de peso lo obtuvo el nivel $1.0 \mathrm{~kg} \mathrm{t}^{-1}$ con 535.70 $\mathrm{g}$, seguido del nivel $1.5 \mathrm{~kg} \mathrm{t}^{-1}$ (476.15 g), nivel $0.50 \mathrm{~kg} \mathrm{t}^{-1}$ (429.87 g) y el testigo con $388.84 \mathrm{~g}$. Estos resultados son similares a los obtenidos por Vásquez y Fernández (2010), que evidenciaron incremento de pesos $516.2 \mathrm{~g}$ (Actigen, $400 \mathrm{~g} \mathrm{t}^{-1}$ ), y $517.0 \mathrm{~g}$ (Actigen $200 \mathrm{~g} \mathrm{t}^{-1}$ ). Igualmente Gómez (2012) a los 42 días de edad de los pollos, observo incrementos de pesos de 603.43 g (Bio-Mos $\left.1 \mathrm{~kg}^{-1}\right)$, y $591.55\left(0,75 \mathrm{~kg}^{-1}\right)$ en dietas balanceadas sobre el rendimiento productivo en pollos Broilers, mayores a los evaluados en la presente investigación.

Tabla 6. Efectos de los niveles de mánanos oligosacáridos en el Incremento de peso en pollo de engorde

\begin{tabular}{|c|c|c|c|c|c|c|}
\hline \multirow{2}{*}{ Semanas } & \multicolumn{4}{|c|}{ Tratamientos } & \multirow{2}{*}{ E.E.M. } & \multirow{2}{*}{$\mathrm{P} \geq$} \\
\hline & T0 & $\mathrm{T} 1$ & $\mathrm{~T} 2$ & $\mathrm{~T} 3$ & & \\
\hline 1 & $118.24 \mathrm{a}$ & $121.36 \mathrm{a}$ & $119.80 \mathrm{a}$ & $119.34 \mathrm{a}$ & 1.29 & 0.7641 \\
\hline
\end{tabular}




\begin{tabular}{ccccccc}
2 & $255.32 \mathrm{a}$ & $247.78 \mathrm{ab}$ & $259.60 \mathrm{a}$ & $231.29 \mathrm{~b}$ & 4.28 & 0.0017 \\
3 & $378.68 \mathrm{a}$ & $401.34 \mathrm{ab}$ & $408.85 \mathrm{~b}$ & $465.64 \mathrm{ab}$ & 7.00 & 0.0001 \\
4 & $508.37 \mathrm{a}$ & $502.37 \mathrm{a}$ & $488.35 \mathrm{a}$ & $486.65 \mathrm{a}$ & 7.40 & 0.3719 \\
5 & $645.97 \mathrm{a}$ & $622.44 \mathrm{a}$ & $596.17 \mathrm{a}$ & $625.49 \mathrm{a}$ & 17.15 & 0.6652 \\
6 & $388.84 \mathrm{~b}$ & $429.87 \mathrm{ab}$ & $535.70 \mathrm{a}$ & $476.15 \mathrm{ab}$ & 16.54 & 0.0066 \\
Total & $2295.42 \mathrm{c}$ & $2325.16 \mathrm{bc}$ & $2409.00 \mathrm{a}$ & $2405.02 \mathrm{~b}$ & 7.34 & 0.0010 \\
\hline
\end{tabular}

Promedios con letras iguales no difieren estadísticamente, según Tukey $(\mathrm{P} \leq 0.05)$. EEM $=$ error estándar de la media; $\mathrm{CV} \%=$ coeficiente de variación; .T0= Testigo; $\mathrm{T} 1=0,5 \mathrm{~kg} \mathrm{t}^{-}$

${ }^{1} \mathrm{MOs} ; \mathrm{T} 2=1 \mathrm{~kg} \mathrm{t}^{-1} \mathrm{MOs} ; \mathrm{T} 3=1,5 \mathrm{~kg} \mathrm{t}^{-1} \mathrm{MOs}$.

\section{Rendimiento a la canal}

El rendimiento a la canal a los 42 días de edad de los pollos de engorde tuvo significancia $(\mathrm{P} \leq 0.05)$ entre los tratamientos, el mejor rendimiento a la canal lo tuvo el nivel $0.50 \mathrm{~kg} \mathrm{t}^{-1}$ (T1) de 75,89\%, Figura 1. Estos resultados son similares a los obtenidos por Vásquez y Fernández (2010), quienes utilizaron Actigen $200 \mathrm{y} 400 \mathrm{~g} \mathrm{t}^{-1}$, alcanzaron rendimiento a la canal de $74.50 \%$ y $73 \%$ a los 42 días de edad de los pollos. Similar estudio realizo Artiga (2002), evaluó cepas de levaduras Safmannan con dosis de 1 $\mathrm{kg} \mathrm{t}^{-1}$ y obtuvo rendimiento a la canal de $72.2 \%$ en pollos de engorde a los 42 días de edad.

Figura 1. Rendimiento a la canal, $\%$

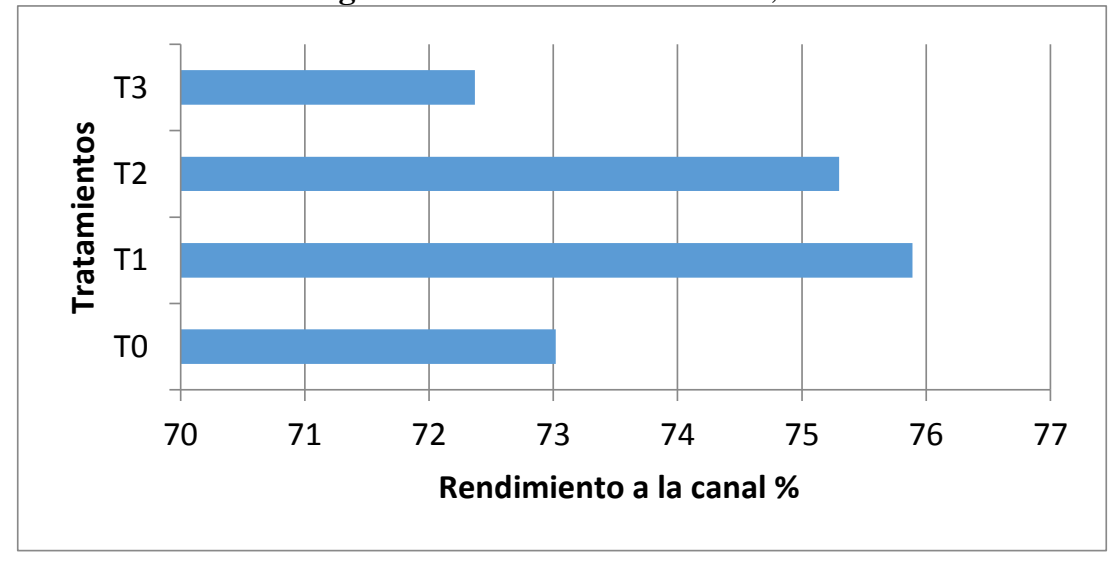

\section{Mortalidad}

El T3 registro el menor porcentaje de mortalidad 2\%, seguido de T0,T1 y T2 con el 3\%. Figura 2. Estos resultados son menores a los obtenidos por Pardo y Gómez (2008) donde evaluaron la adición de mánanos oligosacáridos en el alimento en pollos de engorde de la línea Ross 308 , registraron una mortalidad de $4.19 \%$. En otra investigación realizada por Arce et al. (2005), se registró mortalidad de 5.40\% $\left(1 \mathrm{~kg} \mathrm{t}^{-1}\right), 5 \%(0.5 \mathrm{~kg}$ $\left.\mathrm{t}^{-1}\right)$ y $7.1 \%\left(0.25 \mathrm{~kg} \mathrm{t}^{-1}\right)$ al evaluar efectos de paredes celulares (Saccharomyces cerevisiae) en el alimento en pollos de engorde a los 49 días de edad. 
Por su parte Nicolalde (2009), reporto mortalidad de $1.33 \%$ con la utilización de mánanos oligosacárido $\left(1.0 \mathrm{kgt}^{-1}\right.$ de alimento) y ácido orgánico en los parámetros productivos en pollos de engorde, a los 49 día edad porcentajes menores a los de la investigación realizada.

Figura 2. Mortalidad, \%

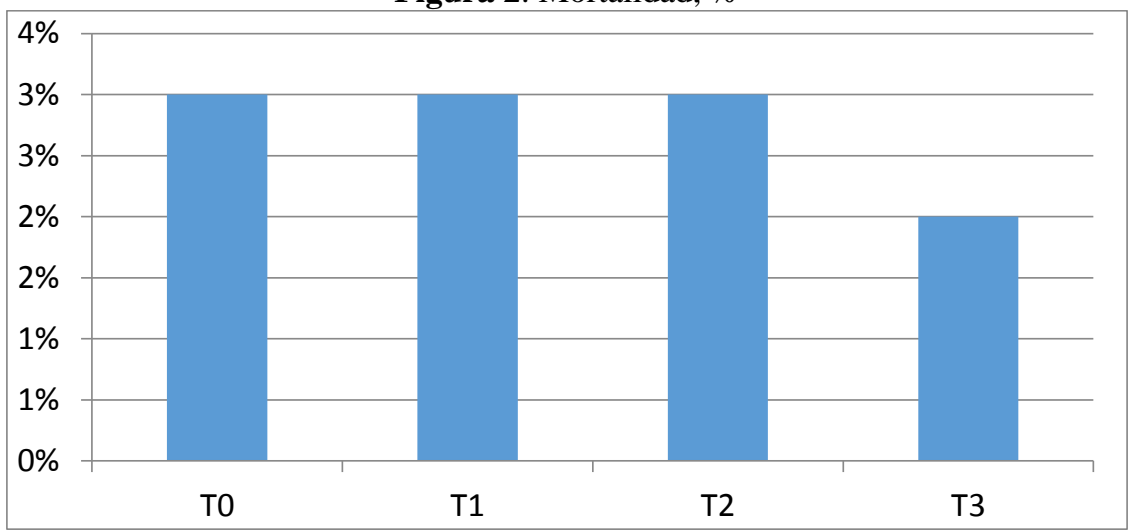

\section{Longitud de las vellosidades intestinales en pollos de engorde}

Las medias genérales de la longitud de las vellosidades intestinales se presentan en la Tabla 7, a los 21 días de edad de los pollos en el yeyuno e íleon no hubo significancia $(\mathrm{P} \geq 0.05)$, pero si se encontró significancia $(\mathrm{P} \leq$ 0.05) en el duodeno. En el yeyuno, e íleon a los 35 días presentaron significancia $(\mathrm{P} \leq 0.05)$, al contrario no hubo en el íleon. Respecto a los 42 días de edad de los pollos las vellosidades en el duodeno presento significancia $(\mathrm{P} \leq 0.05)$, pero no a nivel del yeyuno e íleon $(\mathrm{P} \geq 0.05)$. A los 21 días, el duodeno con nivel $1.5 \mathrm{~kg} \mathrm{t}^{-1}$ de MOs, alcanzo mayor longitud de 1939.74 (um), yeyuno a los 35 días con nivel $0.5 \mathrm{~kg} \mathrm{t}^{-1}$, la longitud fue de 1594.03 (um), Íleon a los 42 día obtuvo una longitud 1196.93 (um) con nivel $0.5 \mathrm{~kg} \mathrm{t}^{-1}$. Estos resultados son diferentes a los obtenidos por Nicoletti, Quintana, Terraes y Kuttel (2010), los que evaluaron parámetros productivos y morfológicos en pollos parrilleros suplementados con ácidos orgánicos y levaduras $\left(1.5 \mathrm{~kg}^{-1}\right)$, con valores para la altura de las vellosidades (um) en duodeno a los 21 días de (1669.8) y 35 días de 1923.8, así mismo para el yeyuno y Íleon a los 21 de 899.4 y a los 35 con 1313.2 días. Sonmez y Eren (1999); Iji (2001); Baurhoo, Phillip y Ruiz (2007), observaron un incremento de la longitud de las vellosidades en pollos alimentados con pienso suplementado con MOs (mánanos-oligosacárido en razón de $1 \mathrm{~g} \mathrm{~kg}^{-1}$ ). Iji (2001) demostró aumentos en la altura de las vellosidades del yeyuno de pollos alimentados con mánanos oligosacáridos obtenidos de levaduras (BioMos ${ }^{\circledR} 5 \mathrm{~g} \mathrm{~kg}^{-1}$ a los 28 días de edad). Bradley, Savage y Timn (1994) en su ensayos con pollos de engorde, encontraron que la inclusión de levaduras de 
Saccharomyces cerevisiae $\left(0,2 \mathrm{~g} \mathrm{~kg}^{-1}\right)$ en el alimento no tuvo un efecto sobre la altura y amplitud de las vellosidades del íleon.

Tabla 7. Efectos de mananos oligosacáridos (MOs) sobre la altura de las vellosidades intestinales $(\mu \mathrm{m})$ en pollos de engorde.

\begin{tabular}{|c|c|c|c|c|c|c|}
\hline \multirow[t]{2}{*}{$\begin{array}{l}\text { Región } \\
\text { intestinal }\end{array}$} & \multirow[t]{2}{*}{ Días } & \multicolumn{4}{|c|}{ Tratamientos } & \multirow[t]{2}{*}{$\mathrm{P} \geq$} \\
\hline & & T0 & $\mathrm{T} 1$ & $\mathrm{~T} 2$ & $\mathrm{~T} 3$ & \\
\hline \multirow{3}{*}{ Duodeno } & 21 & $1465.60 \mathrm{~b}$ & $1469.70 b$ & $1812.60 \mathrm{a}$ & $1939.74 a$ & 0.0001 \\
\hline & 35 & $1346.13 a$ & $1610.45 \mathrm{a}$ & $1426.08 \mathrm{a}$ & $1483.78 \mathrm{a}$ & 0.1203 \\
\hline & 42 & $1367.73 a$ & $1534.35 \mathrm{a}$ & $1453.75 \mathrm{a}$ & $1569.08 \mathrm{a}$ & 0.0568 \\
\hline \multirow{3}{*}{ Yeyuno } & 21 & $1306.08 \mathrm{a}$ & $1295.43 a$ & $1295.43 a$ & $1184.2 \mathrm{~b}$ & 0.0003 \\
\hline & 35 & $488.80 \mathrm{c}$ & $1594.03 \mathrm{a}$ & $1012.03 b$ & $582.73 c$ & 0.0001 \\
\hline & 42 & $1012.58 b$ & $835.13 c$ & $1419.23 a$ & $901.23 c$ & 0.0001 \\
\hline \multirow{3}{*}{ Íleon } & 21 & $848.93 \mathrm{a}$ & $909.20 \mathrm{a}$ & $974.83 a$ & $974.83 \mathrm{a}$ & 0.0344 \\
\hline & 35 & $919.58 \mathrm{ab}$ & $1022.23 \mathrm{a}$ & $787.90 \mathrm{~b}$ & $911.88 \mathrm{ab}$ & 0.0081 \\
\hline & 42 & $651.38 \mathrm{c}$ & $1196.93 a$ & $1010.48 b$ & $710.75 \mathrm{c}$ & 0.0001 \\
\hline
\end{tabular}

Promedios con letras iguales no difieren estadísticamente, según Tukey $(\mathrm{P} \leq 0.05)$. EEM $=$ error estándar de la media; $\mathrm{CV} \%=$ coeficiente de variación; .T0 0 Testigo; $\mathrm{T} 1=0,5 \mathrm{~kg} \mathrm{t}^{-}$

${ }^{1} \mathrm{MOs} ; \mathrm{T} 2=1 \mathrm{~kg} \mathrm{t}^{-1} \mathrm{MOs} ; \mathrm{T} 3=1,5 \mathrm{~kg} \mathrm{t}^{-1} \mathrm{MOs}$.

\section{Ancho micras $(\mu \mathrm{m})$ de las vellosidades intestinales}

En el ancho de las de vellosidades intestinales hubo significancia $(\mathrm{P} \leq$ 0.05 ) con los niveles de mánanos oligosacáridos tanto en duodeno, yeyuno y Íleon a $\operatorname{los} 21,35$ y 42 días de edad de los pollos, entre los tratamientos Tabla 8. A los 21 días el ancho de las vellosidades del duodeno fue mayor $(\mathrm{P} \leq 0.05)$ con nivel $1.5 \mathrm{~kg} \mathrm{t}^{-1}$ (326.03 um), yeyuno nivel $1 \mathrm{~kg} \mathrm{t}^{-1}$ (220.08 um), íleon con nivel $1.5 \mathrm{~kg} \mathrm{t}^{-1}(262.18 \mathrm{um})$. Estos resultados son similares a los obtenidos por Arce et al (2008), evaluaron paredes celulares de Saccharomyces cerevisiae (PcSc) $500 \mathrm{~g} \mathrm{t}^{-1}$ en pollos de engorde a los 21 día de edad, sobre la variable productiva y longitud y ancho de las vellosidades intestinales a los 10 y 21 días de edad, observando ancho de vellosidades de 307, 394, 470, 420 (um). Además indica que la edad del ave es determinante en las evaluaciones intestinales, a mayor edad, mayor amplitud, número y área de las vellosidades.

Tabla 8. Efectos de los mánanos oligosacáridos (MOs) sobre el ancho de las vellosidades intestinales en pollos Broilers.

\begin{tabular}{ccccccc}
\hline \multirow{2}{*}{$\begin{array}{c}\text { Región } \\
\text { Intestinal }\end{array}$} & Días & \multicolumn{4}{c}{ Tratamientos } & \multirow{2}{*}{$\mathrm{P} \geq$} \\
\cline { 3 - 6 } & & $\mathrm{T} 0$ & $\mathrm{~T} 1$ & $\mathrm{~T} 2$ & $\mathrm{~T} 3$ & \\
\cline { 3 - 6 } Duodeno & 21 & $276.93 \mathrm{a}$ & $167.00 \mathrm{~b}$ & $187.63 \mathrm{~b}$ & $326.03 \mathrm{a}$ & 0.0046 \\
& 35 & $119.53 \mathrm{~b}$ & $144.53 \mathrm{ab}$ & $239.30 \mathrm{a}$ & $204.68 \mathrm{ab}$ & 0.0112 \\
& 42 & $148.38 \mathrm{a}$ & $163.57 \mathrm{a}$ & $152.63 \mathrm{a}$ & $198.58 \mathrm{a}$ & 0.1084
\end{tabular}




\begin{tabular}{ccccccc} 
& 21 & $118.50 \mathrm{~b}$ & $163.68 \mathrm{ab}$ & $220.08 \mathrm{a}$ & $167.68 \mathrm{ab}$ & 0.0026 \\
Yeyuno & 35 & $137.63 \mathrm{a}$ & $142.35 \mathrm{ab}$ & $164.18 \mathrm{a}$ & $108.58 \mathrm{~b}$ & 0.0578 \\
& 42 & $174.35 \mathrm{a}$ & $95.50 \mathrm{~b}$ & $142.60 \mathrm{ab}$ & $98.78 \mathrm{~b}$ & 0.0111 \\
\multirow{3}{*}{ Íleon } & & & & & & \\
& 21 & $151.75 \mathrm{~b}$ & $152.23 \mathrm{~b}$ & $216.35 \mathrm{ab}$ & $262.18 \mathrm{a}$ & 0.0006 \\
& 35 & $122.23 \mathrm{a}$ & $137.70 \mathrm{a}$ & $154.45 \mathrm{a}$ & $118.40 \mathrm{a}$ & 0.5863 \\
& 42 & $123.33 \mathrm{a}$ & $132.60 \mathrm{a}$ & $90.30 \mathrm{a}$ & $84.00 \mathrm{a}$ & 0.0870
\end{tabular}

Promedios con letras iguales no difieren estadísticamente, según Tukey $(\mathrm{P} \leq 0.05) . \mathrm{EEM}=$ error estándar de la media; $\mathrm{CV} \%=$ coeficiente de variación; .T0 0 Testigo; $\mathrm{T} 1=0,5 \mathrm{~kg} \mathrm{t}^{-}$

${ }^{1} \mathrm{MOs} ; \mathrm{T} 2=1 \mathrm{~kg} \mathrm{t}^{-1} \mathrm{MOs} ; \mathrm{T} 3=1,5 \mathrm{~kg} \mathrm{t}^{-1} \mathrm{MOs}$.

Rendimiento económico de pollos Broilers con la adición de mananos oligosacáridos en el alimento comercial

El beneficio de cada tratamiento se muestra en la Tabla 9, el rendimiento económico fue mejor para el tratamiento con nivel de $1 \mathrm{kgt}^{-1}$ (T2). los resultados obtenidos en la investigación, difieren a los obtenidos por Gómez (2012), evaluó mánanos oligosacáridos a dosis de $0,75 \mathrm{~kg}^{-1}$ y 1 $\mathrm{kg}^{-1}$ y determino que con $0,75 \mathrm{~kg}^{-1}$ de mánanos oligosacáridos adicionado en el alimento comercial registro mayor beneficio neto. Igualmente Piad y Reyes (2013) obtuvieron mayor beneficio neto, con la adición de $0.75 \mathrm{~g} \mathrm{~kg}^{-1}$ de paredes celulares Saccharomyces cerevisiae (PCL-Glucanos) en el comportamiento productivo en polos de engorde. Por otra Nicolalde (2009) obtuvo beneficio económicos de 1.17 dólares por pollo producido al evaluar mánanos oligosacáridos y ácido orgánico de $1.0 \mathrm{~g} \mathrm{~kg}^{-1}$ de alimento en pollos a los 49 días de edad, menores a los evaluados en la investigación realizada.

Tabla 9. Costo/Beneficio en pollos Broilers con diferente niveles de MOs

\begin{tabular}{ccccc}
\hline \multirow{2}{*}{ Ítem } & \multicolumn{3}{c}{ Tratamientos } \\
\cline { 2 - 5 } & 2342.93 & 2380.18 & 2455.48 & 2392.57 \\
\hline Peso promedio final(gr/ave & 60.00 & 60.00 & 60.00 & 60.00 \\
Total de pollos inicial & 57.00 & 57.00 & 57.00 & 58.00 \\
total de pollos final & 5.00 & 5.00 & 5.00 & 3.30 \\
Mortalidad (\%) & 4342.63 & 4304.57 & 4216.8 & 4254.32 \\
Consumo alimento (g/ave) & 299.84 & 299.19 & 296.19 & 298.86 \\
Total de egresos & & & & \\
Ingresos & 133.54 & 135.66 & 139.65 & 138.62 \\
Kilogramos de pollos vendidos & 2.75 & 2.75 & 2.75 & 2.75 \\
Precio de venta (kg) & 367.24 & 373.07 & 384.04 & 381.21 \\
\hline Ingreso por venta de pollos $(\$)$ & 1.22 & 1.25 & 1.30 & 1.28 \\
\hline Costo/Beneficio & & &
\end{tabular}

\section{Conclusiones y Recomendaciones}

La adición de mánanos oligosacáridos (MOs) en el alimento balanceado comercial, el nivel de $1 \mathrm{~kg} \mathrm{t}^{-1}$, mejoró los parámetros 
productivos (peso corporal, consumo de alimento, conversión alimenticia y porcentaje de mortalidad) frente al tratamiento testigo. El porcentaje de mortalidad fue menor con el nivel de $1,5 \mathrm{~kg} \mathrm{t}^{-1}$ (T3). Con respecto al rendimiento a la canal el nivel de $0.5 \mathrm{~kg} \mathrm{t}^{-1}$ (T1) de MOs tuvo mejor rendimiento $(75.3 \%)$.

La niveles de mánanos oligosacáridos aumento significativamente $(\mathrm{P} \geq 0.05)$ la longitud y anchura de las vellosidades intestinales, a los 21 días de edad de los pollos, el duodeno alcanzó mayor altura (micras) y anchura, en relación a los 35 y 42 días entre los tratamientos.

El rendimiento económico fue mejor con la adición de $1 \mathrm{kgt}^{-1} \mathrm{de}$ mánanos oligosacáridos (MOs) en el alimento balanceado comercial (T2). Esta investigación nos sirve de guía para seguir realizando investigaciones sobre la utilización de los mánanos oligosacáridos, en la producción de aves teniendo en cuenta los beneficios económicos.

Incluir mánanos oligosacáridos (MOs), en una proporción de $1 \mathrm{~kg} \mathrm{t}^{-}$ 1 en el alimento, en las fases de crecimiento y acabado

Evaluar el comportamiento productivos en pollos de engorde con la adición de mánanos oligosacáridos en el agua con diferente niveles.

Estudiar los parámetros productivos con la inclusión de levaduras de cerveza Saccharomyces Cerevisiae en otras especies pecuarias como: Bovinos, porcinos, equinos, cuyes, conejos, etc.

Realizar un nuevo trabajo investigativo donde se evalué las alteraciones en la mucosa intestinal en aves de engorde y postura, con la adición de mánanos oligosacáridos en el alimento y agua.

Emplear mánanos oligosacáridos (MOs) con niveles de $1.0,1.5 \mathrm{~kg} \mathrm{t}^{-1}$ en dietas para pollos de engorde, se obtiene mayor rendimiento económico.

\section{References:}

1. Arce, J; Avila, E; Coello, C;. (2008). Comportamiento productivo y cambios morfologicos en vellosidades intestinales del pollo de engordea 21 dias de edad con el uso de paredes celulares del sacchoromyces cerevisiae. Veterinaria Mexico, Vol. 39 No 2, Pag 15 .

2. Baurhoo, B; Philip, L; Ruiz, C. (2007). Effects of purifield lignin and mannan oligosaccharidaes on intestinal integrity and microbial populations in the ceca and litter of Broilers chickens. Pout. Sci, 86:1070-1078.

3. Bayley, R. (2013). Salud intestinal, aves domesticas. Disponible:http:// www.aviagen.com.

4. Benitez, V., \& Reina, R. (2007). Evaluacion de oligosacaridos mánanoss: Bio-Mos y Safmannan en la productividad de pollos. Honduras: Tesis de pregrado. 
5. Bradley, GL; Savage, TF; Timm, KL. (1994). The effects of supplementing diets with Saccharomyces cerevisiae var.Boulardii on male poultry perfomance and ileal morphology. Poultry Science, 73:1766-1770.

6. CONAVE. (2012). Corporacion Nacional de Avicultores del Ecuador. Recuperado el 4 de MAYO de 2015, de Disponible: http://avicultura2015/conave/

7. Duque, J. (2010). Planificacion de la produccion avicola. Quito, Ecuador: Tesis de pregrado.

8. Gaggia, F; Marttarelli, P; Biavat, B;. (2010). Probiotics and prebiotics in animal feeding for safe food production. Inf.J. Microbial, 141: 1528.

9. Gomez, S. (2012). Evaluacion de dos niveles de oligosacaridos mánanoss como aditivo natural en dietas balanceadas sobre el rendimiento productivo en pollos de engorde en las tres fases de desarrollo en el canton Babahoyo. Babahoyo, Ecuador: Universitadad Tecnica de Babahoyo.

10. Gutierrez, L., Montoya O., \& Velez, J. (Enero-junio de 2013) Probioticos: una alternativa de producción limpia y de reemplazo a los antibioticos promotores de crecimiento en la alimentación animal, 134- 146.

11. Iji, P. (2001). The impact of cereal non-starch polysaccharides in intestinal . Poultry Science journal, 55(4) :375- 87.

12. Lopez, C. (2010). Efecto de uso de acido organico en la nutricionde aves. Congreso naciona lde nutricion animal. Mexico.

13. Narvaez, C. (2008). Guia practica de Avicultura (1 ed.). Quito, Ecuador: pag 15 .

14. Newman, K. (2002). Como funcionan los oligosacaridos en la produccion animal. Feeding Times, 7(1): 3-5.

15. Nicoletti, D., Flores, C., Terraes , J., \& Kuttel, J. (2010). Paramtros productivocs y Morfologicos en pollos parrilleros suplementados con acido organico y levadura. Sitio Arjentino de produccion animal, 21(1), 23-24.

16. Ortiz, A. (25 de Octubre de 2005). Salud intestinal. Ajuste de dietas. Recuperado el 20 de Agosto de 2014, de Avicultura/ Articulo tecnico / Sanidad: http://www.engormix.com/MAavicultura/sanidad/articulos/salud-intestinal-ajuste-dietas-t831/165p0.htm

17. Pardo, M., \& Gomez, J. (2008). Utilizacion de oligosacaridos mánanoss (BioMos) en produccion de pollos de engorde de la linea Ross en Arbelaez y Silvana. Bogota, Colombia: Tesis.Univeridad de la Salle. 
18. Sonmes, N., \& Eren , M. (1999). Effects of supplementation of zinc bacitracin, manna oligosaccharides and probiotic into the Broilers feed on morphology ofthe small intestine. DergisiUludag Univ, 18: $125-138$.

19. Torrealba, H. (2007). Estrategias alimenticias que influyen la microflora bacteriana. Alltech- Ecuador.

20. Vasquez, A., \& Fernandez, 0. (2010). Efectos de Actigen en lasdietas de pollos de engorde sobre el rendimiento, inmunidad e integridad intestinal. Honduras: Zamorano,tesis de pregrado.

21. Waldroup, P; Fristtis, C; Oviedo, E;. (2003). Copmparison of Biomos and antiotiic Feeding program in Broilers Diets Containing Copper Sulfate. Inter. Jour. Poultry. Science, 2: 28-31.

22. Winder, G. (2004). Factores que afectan la Competitividad del sector avicola en America Latina. Agronegocios, 6, 1-10.

23. Zoetis, C. (2013). Integridad intestinal. Avicultura $m x$, Disponible : www.avicultura.com.mx. 